Cahiers $d u$ MONDE RUSSE

\section{Cahiers du monde russe}

Russie - Empire russe - Union soviétique et États indépendants

$52 / 4 \mid 2011$

Varia

\title{
Nikolai Krementsov, A Martian Stranded on Earth
}

\section{Gregory Dufaud}

\section{OpenEdition \\ Journals}

Édition électronique

URL : http://journals.openedition.org/monderusse/7602

DOI : $10.4000 /$ monderusse. 7602

ISSN : $1777-5388$

Éditeur

Éditions de l'EHESS

\section{Édition imprimée}

Date de publication : 20 décembre 2011

Pagination : 716-718

ISBN : 978-2-7132-2353-2

ISSN : $1252-6576$

\section{Référence électronique}

Gregory Dufaud, « Nikolai Krementsov, A Martian Stranded on Earth», Cahiers du monde russe [En ligne], 52/4 | 2011, mis en ligne le 29 novembre 2012, Consulté le 21 septembre 2020. URL : http:// journals.openedition.org/monderusse/7602 ; DOI : https://doi.org/10.4000/monderusse.7602

Ce document a été généré automatiquement le 21 septembre 2020.

〔c École des hautes études en sciences sociales 


\title{
Nikolai Krementsov, A Martian Stranded on Earth
}

\author{
Gregory Dufaud
}

\section{RÉFÉRENCE}

Nikolai KREMENTSOV, A Martian Stranded on Earth. Alexander Bogdanov, Blood

Transfusions, and Proletarian Science. Chicago : The University of Chicago Press, 2011, $175 \mathrm{p}$.

1 Centré autour de la figure d'Alexandr Bogdanov, le nouvel ouvrage de Nikolai Krementsov, s'il est court, n'en est pas moins passionnant. Ni véritable biographie, ni monographie au sens strict, il chemine entre ces deux genres afin de décrire, à travers sept chapitres, ce qui était qualifié de « science du sang » dans les années vingt. À partir d'un corpus documentaire constitué d'archives et d'imprimés, l'auteur montre comment celle-ci a été pensée, puis promue avant d'être institutionnalisée malgré l'absence de légitimité scientifique de Bogdanov dont le projet de «collectivisme physiologique » ne survit pas à sa disparition en 1928 à cause d'une réaction de rejet provoquée par un échange sanguin: une forme de transfusion où les deux personnes sont tout à la fois donneurs et receveurs.

Qui est donc ce personnage qui développe une méthode d'échange de sang dont il veut faire un instrument de régénération et de refondation sociales? Il n'est autre que Aleksandr Aleksandrovič Malinovskij, un des principaux dirigeants bolcheviks au début du siècle et grand rival de Lenin à la tête du parti. Leur affrontement, on le sait, aboutit à la victoire de Lenin. Sous le pseudonyme de Bogdanov, Malinovskij se détourne alors de la vie politique pour se consacrer à l'écriture et à la science-fiction. En 1908, il publie un premier roman qui expose sa vision du socialisme: Krasnaja zvezda ${ }^{1}$ L'ouvrage développe deux idées-forces. La première présente la science dans la construction de la société socialiste comme seule susceptible de vaincre les obstacles permettant d'y parvenir. La seconde use de la métaphore du collectivisme dans la société martienne, si 
prégnant que les différences physiques et sexuelles ont progressivement été gommées. Science et collectivisme sont connectés par le biais de la transfusion sanguine qui prolonge la vie des habitants de Mars jusqu'à deux cents ans. La transfusion aurait pu n'être qu'un motif littéraire, mais la fiction présente ce que Bogdanov imagine comme outil réel de transformation sociale: elle participe de sa théorisation. Bogdanov développe son programme à partir de 1910 dans divers écrits grâce aux connaissances en biologie qu'il a acquises. Ce faisant, il entend fonder une science prolétarienne dont la Russie serait le laboratoire. La transfusion sanguine fait à ce moment-là encore l'objet de fortes résistances en Europe. Aussi les recherches dans ce domaine sont-elles très limitées. En Russie, la première transfusion est réalisée en 1919 par Vladimir Šamov, un jeune médecin de Saint-Pétersbourg qui avait pu observer lors d'un séjour comment on la pratiquait aux États-Unis avant la guerre. Des travaux sont dès lors conduits sous sa tutelle et la technique se diffuse progressivement.

3 Au moment du renversement du tsarisme, Bogdanov ne participe pas aux événements révolutionnaires et refuse même les fonctions qu'on lui propose. Toutefois, il s'investit dans le Proletkult dont il est membre de la direction. Il enseigne aussi à l'Académie socialiste et poursuit parallèlement son activité de romancier. Lorsque le proletkult fait l'objet de critiques, Bogdanov abandonne ses responsabilités et accepte de prendre part à un voyage à Londres où il découvre l'ouvrage de Geoffrey Keynes, Blood Transfusion, dans lequel l'auteur relate son expérience de médecin militaire pendant la Grande Guerre. À son retour à Moscou, Bogdanov rassemble autour de lui un cercle étroit d'amis qui se réunit régulièrement et en secret pour étudier les procédures de transfusion sanguine. Après plusieurs mois, et aussi quelques démêlés avec la police politique, lesquels affectent sa santé, il expérimente le premier échange sanguin en février 1924. D'autres s'ensuivent entre les membres du groupe, jusqu'à ce que Leonid Krasin, extérieur à ce cercle, mais ancienne connaissance de Bogdanov, souffrant d'anémie, sollicite son aide. La transfusion est un succès, la santé de Krasin s'améliorant. Peu de temps après, en décembre 1925, Stalin convie Bogdanov à un entretien qui porte sans doute, même si aucune source ne vient l'attester, sur le décès récent de Mikhail Frunze. La santé des vieux bolcheviks et des hauts responsables du pays fait l'objet de vives préoccupations. Depuis la mort de Lenin, diverses mesures ont été prises pour les préserver, sans grands résultats. En février 1926, tandis que les principaux dignitaires du régime sont soumis à une visite médicale obligatoire, Valerian Kujbyšev approuve la fondation d'un institut de transfusion sanguine. Placé sous la direction de Bogdanov, sis dans le palais Igumnov' ${ }^{2}$, l'institut procède à partir de mai à des transfusions d'officiels, plutôt de second rang.

4 Ayant obtenu la création d'un établissement unique en son genre, Bogdanov s'emploie à faire reconnaître ses innovations dans une spécialité où il ne bénéficie, au fond, d'aucune espèce d'autorité. Durant l'été et l'automne 1926, il s'attelle à la rédaction d'un livre, La lutte pour la viabilité, qui présente la conception de la science prolétarienne qu'il développe depuis presque vingt ans. Bogdanov situe son travail dans le domaine de la sénescence dont Charles-Édouard Brown-Sequard, successeur de Claude Bernard à la chaire de médecine expérimentale du Collège de France, est la figure tutélaire. De nombreuses recherches sont poursuivies sur le rajeunissement en Europe depuis la fin du XIX ${ }^{e}$ siècle et rien qu'en Russie, entre 1923 et 1925, une douzaine d'ouvrages et une centaine d'articles sont publiés sur ce thème. Bogdanov propose de remplacer les notions de «jeunesse » et de "vieillesse » par celle de «viabilité » qui repose sur deux composantes: la quantité d'énergie dont dispose un organisme et la 
structure qui détermine la distribution des éléments le constituant. L'équilibre des diverses parties de l'organisme serait alors le résultat de la régulation de l'ensemble. Pour restaurer une viabilité déclinante, Bogdanov avance la solution de l'échange sanguin. Le sang, dont il fait le composant unificateur de l'organisme, serait altéré par l'environnement et le purifier grâce à ce moyen permettrait de rétablir l'équilibre perdu. Un premier compte-rendu du livre, élogieux, paraît en décembre 1926 dans les Izvestija. Le caractère laudatif s'explique, selon Nikolai Krementsov, par la relation que Bogdanov entretient avec le rédacteur en chef. Les recensions suivantes sont moins favorables, quand elles ne sont pas franchement critiques. Elles sont écrites par des médecins, les biologistes ignorant tout simplement l'ouvrage. L'échec de Bogdanov à faire admettre ses théories ne l'empêche toutefois pas de poursuivre ses activités jusqu'à sa mort en 1928, laquelle met un terme au projet dont il était le promoteur et le porte-parole.

5 Si Nikolai Krementsov se heurte à plusieurs reprises aux lacunes de la documentation, il parvient néanmoins à produire un récit très riche qui renseigne sur la variété des engagements de Bogdanov. Il montre alors combien il serait fallacieux non seulement de différencier ontologiquement ou chronologiquement le révolutionnaire du romancier ou du scientifique, mais aussi de séparer de manière trop tranchée les registres d'action: parce qu'administré comme une association de proches, l'institut prolonge dans la sphère publique ce qui relevait jadis de la sphère privée. À travers la trajectoire de Bogdanov, l'auteur livre in fine une histoire des possibles et de ses contradictions dans la Russie des années vingt. L'amélioration de la santé de Krasin vaut à Bogdanov, contre toute attente au regard des ennuis jusqu'alors rencontrés, l'attention des responsables du parti qui n'hésitent pas à lui confier un institut malgré l'avis négatif du Commissariat à la santé et des autorités médicales. La fiction devenait ainsi réalité, la science et le socialisme martiens étaient désormais terriens et la Russie réalisait une double révolution, sociale parce qu'aussi scientifique. Pour autant, jamais Bogdanov n'obtint la légitimité scientifique qu'il recherchait en raison d'une conception trop générale des sciences et d'une incapacité à respecter les normes et usages savants.

Bogdanov représente un cas particulier à maints égards. Cette singularité explique certainement pourquoi Nikolai Krementsov n'ose pas les comparaisons et se refuse à interroger la notion même de science. L'ouvrage aurait sans doute alors tout simplement perdu son côté accessible et, c'est suffisamment rare pour y insister, réjouissant? Inutile donc de bouder son plaisir.

\section{NOTES}

1. Krasnaja zvezda, SPb.: Utopija, 1908. Traduit en français, préfacé et annoté par Catherine Prokhoroff, L'étoile rouge, suivi de l'ingérieur Menni : romans utopiques, Lausanne : L'Âge d'Homme, 1985.

2. Situé rue Bol'šaja Jakimanka, il accueille aujourd'hui la résidence de l'Ambassade de France. 